\title{
Beating Heart Modeling and Simulation
}

\author{
$\mathrm{L} \mathrm{Xia}^{1}, \mathrm{MM} \mathrm{Huo}^{2}, \mathrm{X} \mathrm{Zhang}^{3}, \mathrm{Q} \mathrm{Wei}^{4}, \mathrm{~F} \mathrm{Liu}^{1,4}$ \\ ${ }^{1}$ Department of Biomedical Engineering, Zhejiang University, Hangzhou, PR China \\ ${ }^{2}$ Department of Computer Science, Zhejiang University City College, Hangzhou, PR China \\ ${ }^{3}$ Department of Biomedical Engineering, University of Minnesota, Minneapolis, USA \\ ${ }^{4}$ The School of Information Technology and Electrical Engineering, The University of Queensland, \\ Queensland, Australia
}

\begin{abstract}
Most previous electrical heart models were static, not beating ones. In this paper, we present a method of beating heart modeling and ECG simulation. The key step is to construct an electromechanical heart model. The cardiac excitation propagation was simulated by electrical heart model, and the resulted cardiac active forces were used to calculate the ventricular wall motion based on a mechanical heart model. Thus the source-field point relative position changes during cardiac systole and diastole can be obtained, and are employed to calculate body surface ECG based on the electrical heart-torso model. The results showed that compared with simulated ECG based on the static electrical heart model, the simulated ECG based on beating heart model is more accordant with clinical recorded ECG, especially for ST segment and T wave of V1-V6 lead ECG. For slightdegree myocardial ischemia, the $S T$ segment and $T$ wave changes can be observed from the simulated ECG based on beating heart model, while the ST segment and T wave of simulated ECG based on static heart model is almost unchanged compared with normal ECG.
\end{abstract}

\section{Introduction}

Computer modeling of the heart is a useful theoretical tool for the investigation of cardiac physiology and pathology. In the past twenty years, many effective methods have been developed in order to improve the simulation accuracy of the electrical heart model. For example, researchers have attempted to model the heart with smaller units, or considered of the inhomogeneity of the torso and even the myocardial anisotropy in their heart-torso models [1,2]. These models, however, most of them were static, not beating ones. When computing body surface ECG based on quasi-static field theory, the distances between equivalent cardiac dipoles (source points) and body surface (field points) were supposed to be constant during whole cardiac cycle. This maybe reasonable for the simulation of ECG QRS complex, for the ST segment and T wave, however, large system errors could be introduced because of the significant relative position changes of source-field points during cardiac systole and diastole. Therefore, to improve the ECG simulation accuracy, a beating or dynamic heart model should be considered.

To construct a beating or dynamic electrical heart model, the myocardial wall motion information is required. At present, many kinds of medical imaging methods, including MRI, echocardiography and CT, etc., can be used to investigate the cardiac wall motion [3-5]. However, the complexity of left ventricle (LV) motion, the absence of internal landmarks in the myocardium and the influence of the right ventricle (RV) on the LV imply that the real motion trajectories of tissue elements are, at best, difficult to obtain fully from image analysis. Mechanical heart modeling and simulation could be an effective approach to study the cardiac motion. So we proposed such a method of beating heart modeling and ECG simulation: the cardiac excitation propagation was simulated by electrical heart model, and the resulted cardiac active forces were used to calculate the ventricular wall motion based on a mechanical heart model. Thus the source-field point relative position changes during cardiac systole and diastole can be obtained to calculate body surface ECG with an electrical heart-torso model.

\section{Methods}

\subsection{Electromechanical biventricular model construction}

As Fig. 1 showed, the biventricular model was constructed based on our previously developed electrical heart model [6]. Based on CT image data of the human body and heart sections, the electrical heart model was reconstructed as a 3-D array of approximately 65000 cell units spaced $1.5 \mathrm{~mm}$ apart. It has been proved that this 
model is efficient with reasonable accuracy for cardiac electrical property simulation [6]. It is well known that the myocardial fiber structure of the heart plays a critical role in electrical propagation and force production. Myocardial electrical propagation is anisotropic, with the spread of current largest in the direction of the long axis of the fiber. And also, the fiber orientation is an important determinant of myocardial stress and strain. According to the report in the literature [7], we considered the fiber orientation in the biventricular model as follows: in the left and right ventricular free wall, fiber orientation typically varies from $-60^{\circ}$ at the epicardium to $+90^{\circ}$ at the endocardium, whereas in the septal wall the fiber angle ranges from approximately $-90^{\circ}$ at the right ventricular endocardium to around $+80^{\circ}$ at the left ventricular endocardium. At the junction of ventricular free wall and the septum, certain interpolation function was considered to make the junction smoothly.

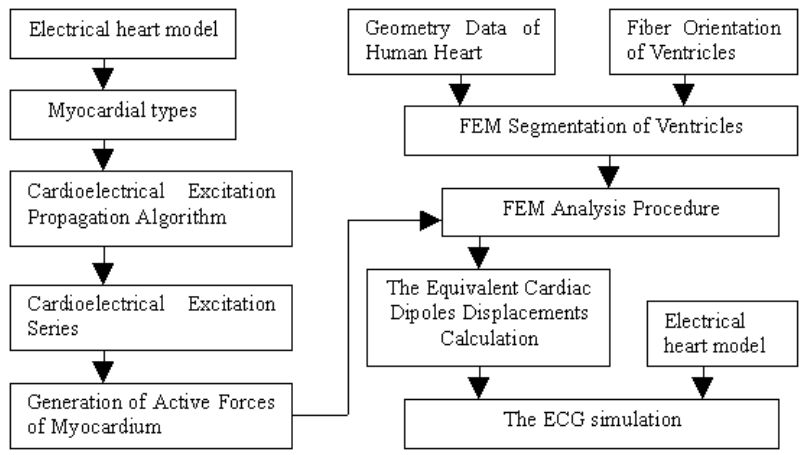

Fig. 1 The construction flow chart of biventricular model.

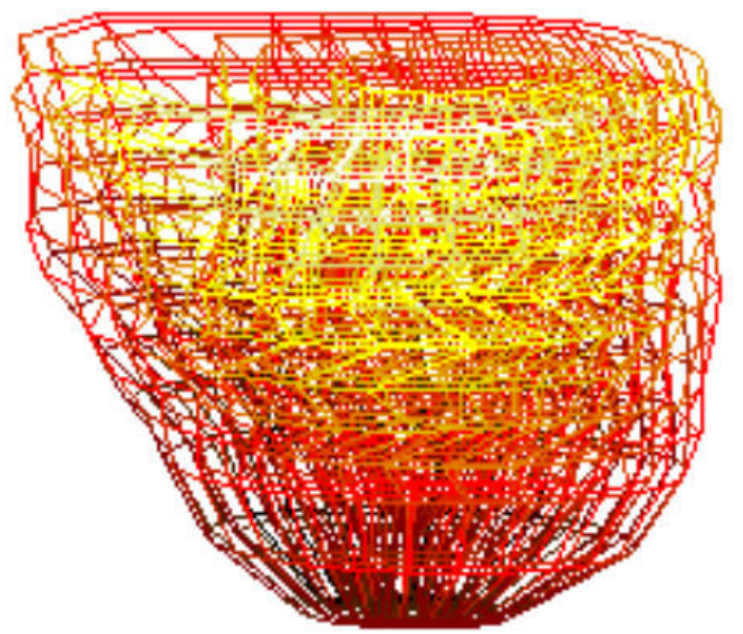

Fig. 2 The grid representation of mechanical biventricular model.

The 3-D finite element mechanical biventricular model was built based on composite theory. 3-D 8-nodes isoparametric element was the basic element, and there were total 13-layer nodes, 1489 brick elements, and 5937 degrees of freedom in this model as shown in Figure 2.

\subsection{Myocardium active forces calculation}

After the cardiac electrical excitation series are determined based on the electrical simulation, we could compute the resultant active forces of myocardium [8].

In the fiber-coordinate system (one axis is chosen to coincide with the local muscle fiber direction, another one is determined by the epicardium surface normal vector), the active force along the fiber might be approximated as:

$$
\sigma_{e}^{\prime}=\left\{\begin{array}{cc}
\sigma_{o}^{\prime} \sin \left(\frac{t-\tau}{T_{e}} \pi\right), & 0 \leq t \leq T_{e} \\
0, & t>T_{e}
\end{array}\right\}
$$

where $t$ is the transient time; $\tau$ is the time lag of active stress, $T_{e}$ is the activation period. $\sigma_{o}^{\prime}$ is the active force of a myocardial fiber and which is a function of fiber length, time $t$, fiber directions, etc.

In the calculation, the stress and strain vector is expressed with respect to two coordinate frames of reference: global coordinate system $X-Y-Z$ and local coordinate system $\xi-\eta-\zeta$. The nodal force equivalent to the active contractile force is calculated based on equation (1) and the isoparametric transformation:

$$
\left\{F_{f}\right\}^{e}=-w \sum_{l=1}^{L_{e}} \int_{\xi_{l-1}}^{\xi_{l}} \int_{-1}^{1} \int_{-1}^{1}[B]^{T} T\left\{0,0, \sigma_{e}^{\prime}, 0,0,0\right\}_{l}^{T}|J| d \xi d \eta d \zeta
$$

where $[B]$ is geometric matrix of element, $l, L_{e}$ stand for the number and total number of layers in an element respectively, $T$ is the transformation matrix between the fiber coordinate and global coordinate. $\left\{0,0, s_{e}^{\prime}, 0,0,0\right\}_{l}^{T}$ is the active force row vector in the fiber-coordinate system, $|J|$ is the determinant of the Jacobian matrix. $\xi, \eta, \zeta$ are the local coordinate system with the magnitudes ranging from -1 to $1 ; \xi^{l}$ and $\xi^{l-1}$ represents individually the coordinate values along $\xi$ direction on the front-side and back-side of layer $l$ in an element. $\xi^{l-1}=-1$ when $l=1$ and $\xi^{k}=1$ when $l=L_{e} . W$ is a myocardial ischemic factor. It represents the ability of the tissue to generate active force and varies between 0 (completely ischemic) and 1 (normal).

\subsection{Calculation of the equivalent cardiac dipole displacements}

The calculation of the equivalent cardiac dipole displacements is based on strain computation of cardiac wall. Strain analysis is a method to describe the internal deformation of a continuum body. It is a useful tool to study and quantify myocardial deformation. The Lagrangian strain $\mathrm{E}$ is used to describe systolic deformation in a region surrounding a point in the cardiac wall relative to its initial position at end-diastole. E was calculated from the relationship:

$$
E=1 / 2\left(F^{T} F-I\right)
$$

where the superscript $\mathrm{T}$ represents the matrix transpose, $\mathrm{F}$ 
represents deformation gradient tensor, and I, the identity matrix. The deformation gradient tensor $F$ can be calculated from the relationship:

$$
F_{p q}=\partial x_{p} / \partial X_{q}
$$

where $\mathrm{X}$ and $\mathrm{x}$ represent the initial and final position of a point in the material respectively, the subscripts $\mathrm{p}$ and $\mathrm{q}$ range from 1 to 3 and denote one of the 3D Cartesian coordinates. The tensor, F, includes both the rotation and deformation around a point in the material.

Now, the displacements of each finite element node during whole cardiac cycle can be obtained, and then the isoparametric inverse transformation is used to calculate the equivalent cardiac dipole displacements for body surface ECG simulation. Under an animation display mode of the displacements of heart wall, the heart seems to be "beating".

\subsection{Myocardial ischemia simulation}

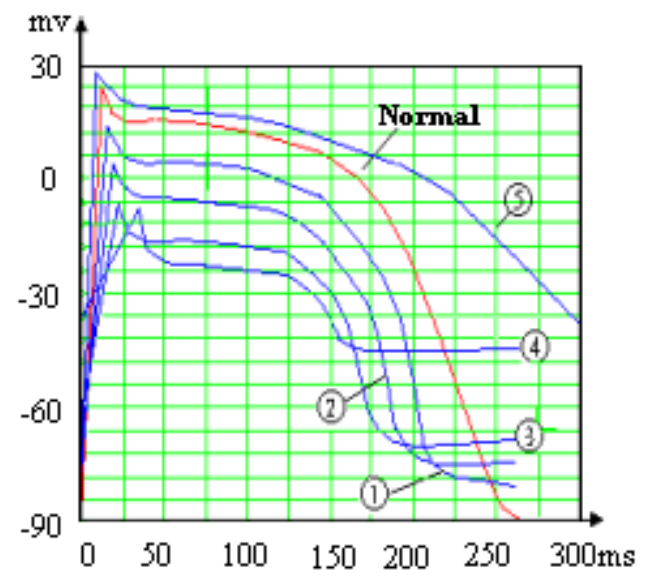

Fig. 3 The action potential waveforms of ischemia myocardium used in the heart-torso model. The injured degree of the muscle become more severe from the action potential curve (1) to (4), and curve (5) is the prolonged action potential waveform caused by ischemia.

Myocardial ischemia or infarction is an imbalance between the supply of oxygenated blood and the oxygen requirements of the myocardium. The ischemia or infarction can alter the myocardial action potentials. The principal changes are decreases in the magnitude of the resting potential and in the action potential duration [9]. Such abnormal action potentials are used in the hearttorso model to simulate myocardial ischemia.

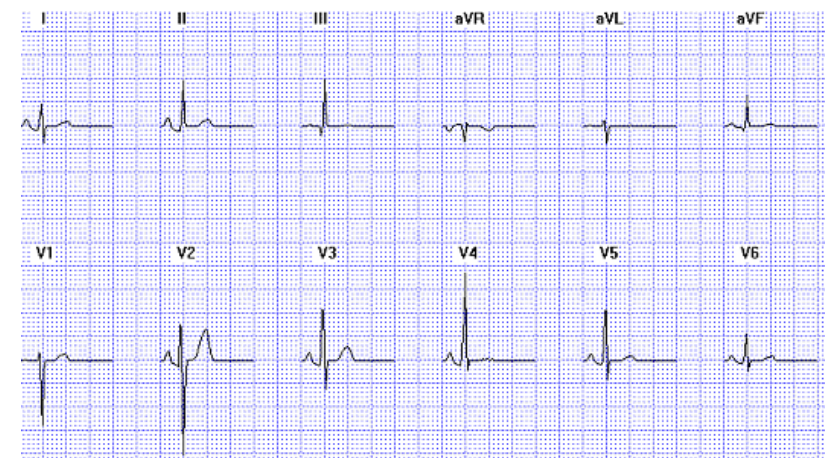

Fig. 4 Normal 12-lead ECG simulation result based on static heart model.

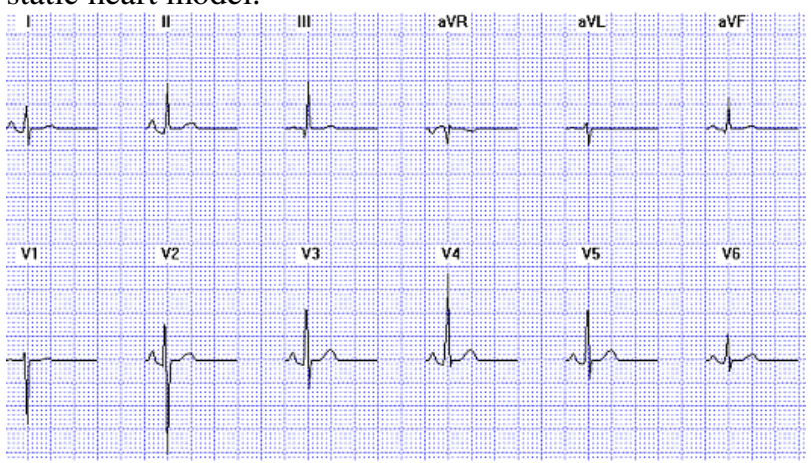

Fig. 5 Normal 12-lead ECG simulation result based on dynamic heart model.

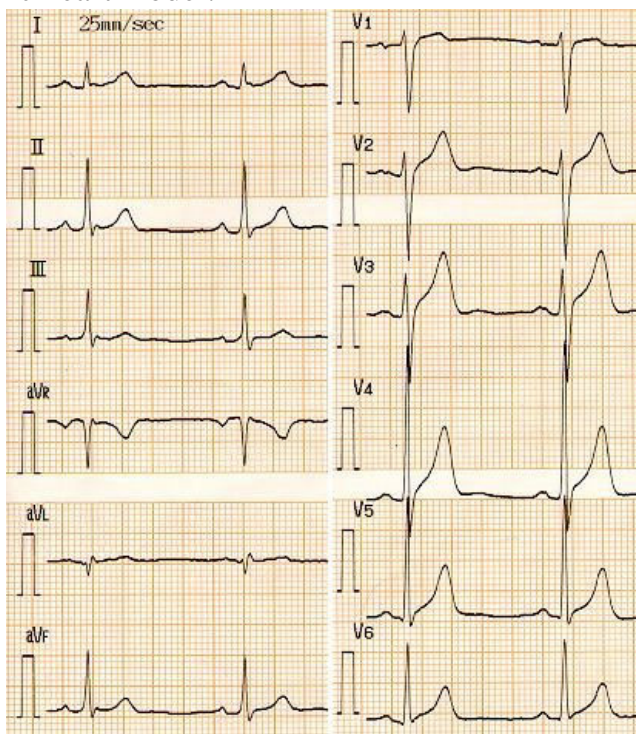

Fig. 6 An example of clinical normal 12-lead ECG record.

\section{Results}

Figure 4 is the simulated 12-lead ECG based on our previous static electrical heart model. Figure 5 shows the 12-lead ECG simulation result using our beating heart model. Figure 6 is an example of clinical 12-lead ECG record. Comparing these figures, we can see that the simulated ECG with beating heart model is more 
accordant with clinical recorded ECG, especially for ST segment and T wave of V1-V6 lead ECG. From Figures 4, 5 we also can see that the QRS complexes are almost unchanged. This is because that the ECG QRS complex corresponds to cardiac isovolumetric contraction phase at which the heart is in its relative static state. While the ECG ST segment and $\mathrm{T}$ wave corresponds to cardiac systole and diastole phases, the relative position changes between heart and body surface are obvious during these periods, and thus result in significant difference of simulated $\mathrm{T}$ wave based on the static heart model and the beating heart model.

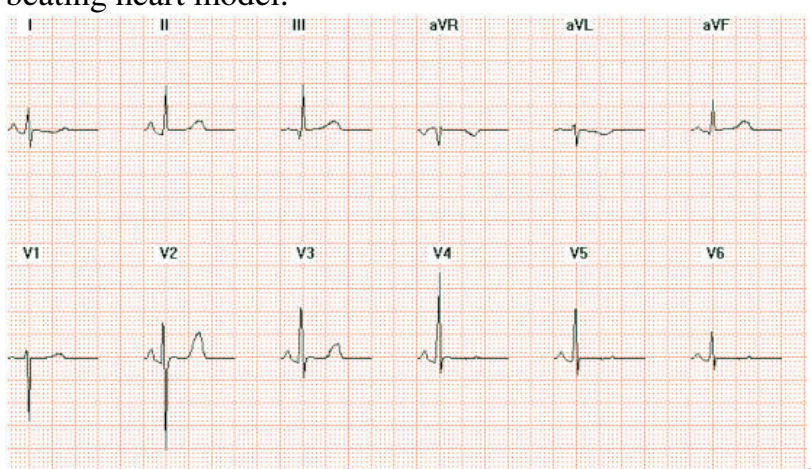

Fig. 7 The simulated 12-lead ECG of anterior wall slight ischemia based on static heart model.

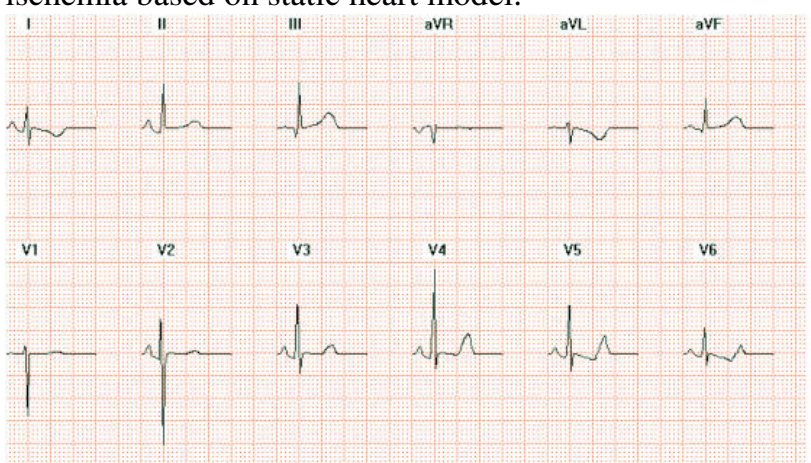

Fig. 8 The simulated 12-lead ECG of anterior wall slight ischemia based on dynamic heart model.

By means of setting abnormal action potentials of heart model cells in some regions, we were able to simulate myocardial ischemia or infarction. Fig. 7 and Fig. 8 show the simulated 12-lead ECG of anterior wall slight ischemia based on static heart model and dynamic heart model, respectively. From Fig. 7 and Fig. 4, we can see that for the anterior wall slight ischemia, the simulated 12-lead ECG with static heart model is almost unchanged compared with the simulated normal 12-lead ECG, only lead I ECG has been altered slightly. From Fig. 8 and Fig. 5 , however, it can be observed that the simulated 12-lead ECG with beating heart model has obvious modifications compared with the simulated normal 12-lead ECG.

\section{Discussion and conclusions}

In this study, a beating heart model was constructed and 12-lead ECG was simulated based on this model. Compared with the simulated ECG based on the static heart model, the simulated ECG based on beating heart model is more accordant with clinical recorded ECG, especially for ST segment and T wave of V1-V6 leads. These simulation results suggest that the beating heart model could generate more accurate ECG signals. For myocardial ischemia or infarction ECG simulation, the beating heart model output is more explainable as the main ECG changes occur at ST segment and $\mathrm{T}$ wave which correspond to cardiac systole and diastole phases.

\section{Acknowledgements}

This work is supported by the National Natural Science Foundation of China (30170243, 30370400), the Foundation for the Author of National Excellent Doctoral Dissertation of P.R. China (1999941), and 973 National Key Basic Research \& Development Program (2003CB716106).

\section{References}

[1] Wei D, Okazaki O, Harumi K, et al. Comparative simulation of excitation and body surface electrocardiogram with isotropic and anisotropic computer heart models. IEEE Trans Biomed Eng 1995; 42: 343-57.

[2] Huiskamp GJ. Simulation of depolarization in a membraneequations-based model of the anisotropic ventricle. IEEE Trans Biomed Eng 1998; 45: 847-55.

[3] Papademetris X, et al. Estimation of 3-D left ventricular deformation from medical images using biomedical models. IEEE Trans Med Imag 2002; 21: 786-800.

[4] Azhari H, Oliker S, et al. Three-dimensional mapping of acute ischemic regions using artificial neural networks and tagged MRI. IEEE Trans Biomed Eng 1996; 43: 619-26.

[5] Linares P, Torrealba V, et al. Deformable model application on segmentation in 3-D echocardiography. Computers in Cardiology 1996; 23: 413-16.

[6] Lu W, Xia L. Computer simulation of epicardial potentials using a heart-torso model with realistic geometry. IEEE Trans Biomed Eng 1996;43:211-7.

[7] Scollan DF, Holmes A, Winslow RL, et al. Histological validation of myocardial microstructure obtained from diffusion tensor magnetic resonance imaging. Am J Physiol 1998; 275: H2308-H2318.

[8] Liu F, Lu W, Xia L. The construction of three-dimensional composite finite element mechanical model of human left ventricle. JSME Int J 2001;44(C1):125-33.

[9] Miller WT, Geselowitz DB. Simulation studies of the electrocardiogram. II. Ischemia and infarction. Circ Res 1978;43:315-23.

Address for correspondence: Ling Xia BME -Zhejiang University, Hangzhou 310027, PR China E-mail:xialing@hzcnc.com. 